\title{
Morus alba Accumulates Reactive Oxygen Species to Initiate Apoptosis via FOXO-Caspase 3-Dependent Pathway in Neuroblastoma Cells
}

\author{
Young Hwi Kwon ${ }^{1,4}$, Kausik Bishayee ${ }^{1,4}$, Md. Ataur Rahman', Jae Seung Hong', Soon-Sung Lim ${ }^{3}$, and \\ Sung-Oh Huh"*
}

\begin{abstract}
Morus alba root extract (MARE) has been used to treat hyperglycaemic conditions in oriental medicine. Here, we studied whether MARE possesses a cytotoxic effect on neuroblastoma. To check the cytotoxicity generated by MARE was whether relatively higher against the cancer cells rather than normal cells, we chose a neuroblastoma cell line (B103) and a normal cell line (Rat-2). A CCK assay revealed that MARE $(10 \mu \mathrm{g} / \mathrm{ml})$ reduced cell viability to approximately $60 \%$ compared to an untreated control in B103 cells. But in Rat-2 cells, MARE induced relatively lower cytotoxicity. To investigate the mechanisms underlying the cytotoxic effect of MARE, we used flow cytometry combined with immunoblot analyses. We found that MARE-treatment could accumulate ROS and depolarize mitochondria membrane potential of B103 cells. Further treatment with MARE in B103 cells also could damage DNA and induce apoptosis. An expression study of p-Akt also suggested that there was a reduction in cellular proliferation and transcription along with the process of apoptosis, which was further evidenced by an increase in Bax and cleaved-caspase 3 activity. Together, our findings suggest that MARE produces more cytotoxicity in cancer cells while having a relatively attenuated effect on normal cells. As such, MARE may be a safer option in cancer therapeutics, and it also shows potential for the patients with symptoms of hyperglycemia and cancer.
\end{abstract}

\section{INTRODUCTION}

The occurrence of childhood and adolescent cancer is rare, but

${ }^{1}$ Department of Pharmacology, College of Medicine, Institute of Natural Medicine, ${ }^{2}$ Department of Physical Education, ${ }^{3}$ Department of Food Science and Nutrition, College of Natural Science, Hallym University, Chuncheon 200-702, Korea, ${ }^{4}$ These authors contributed equally to this work.

${ }^{*}$ Correspondence: s0huh@ hallym.ac.kr

Received 5 February, 2015; accepted 10 March, 2015; published online 3 July, 2015

Keywords: cytotoxicity, intrinsic cell death pathway, neuroblastoma, proliferation, reactive oxygen species the overall incidence of childhood cancer burden is increasing progressively. The orthodox medicines and treatments do not bring any curative to the neuroblastoma patients. Neuroblastoma is a type of childhood cancer where the malignancy develops in immature nerve cells (Friedman and Castleberry, 2007). It commonly arises in and around the adrenal glands (Nuchtern, 2006), which have similar origins to nerve cells and in the abdomen, chest, neck, and spine (Fish and Grupp, 2008). Currently, chemotherapy and radiation therapy are the most commonly used therapeutic approaches against neuroblastoma (Haase et al., 1999; Johnson et al., 2007). These non-targeted therapeutic approaches have severe side-effects because they target and destroy all rapidly growing cells (Lastowska et al., 2001). It is also crucial to find an inexpensive treatment so that it can be accessible to a greater number of individuals. Ancient medical literature reports the use of natural, plant-derived products, which have been found to be beneficial to cancer treatment, such as green tea (which contains epigallocatechin-3-gallate) (Bengmark et al., 2009; Mondal et al., 2012) and turmeric (which contains quercetin and berberine) (Mantena et al., 2006). In this point of time, there is a need to evaluate the natural edible plant products and/or extract against the deadly disease cancer, to ease from the unwanted side effect.

Complementary and alternative medicine (CAM) is becoming increasingly popular particularly in oncology, which often applies various CAMs to give patients a better quality of living by alleviating their suffering (Chakraborty et al., 2013). In Chinese medical treatment, mulberry extract has been traditionally used in the treatment of diabetic nephropathy and is popular for its antiadipogenic and anti-hyperglycemic effects (Gupta and Patel, 2013; Wang et al., 2014). Insulin resistance has been shown to be improved by treatment with mulberry (Ren et al., 2015). Other than that, MARE displays a profound anti-inflammatory effect on macrophages: a study has shown that LPS-induced inflammation can be reduced in RAW 264.7 cells through ERK $1 / 2$ activation in a ROS-dependent process (Eo et al., 2014). In MCF-7 cells, MARE treatment reduced the viability of cancer cells and activated JNK1/2 pathway to induce apoptosis (Choi et al., 2013). Albanol $\mathrm{A}$, isolated from mulberry root, has been shown to promote apoptosis in leukaemia cells through disrupting Bax/Bcl-2 homeostasis and activating caspase 2 (Kikuchi et al., 2010). Studies also showed that geranylated flavonoids isolated from mulberry root possess a cytotoxic effect on THP-1 monocytic leukaemia cells (Smejkal et al., 2010). Another study on leukae- 
mia and melanoma showed that the water extract of mulberry root could disrupt the microtubule assembly of cancer cells; however, it could not arrest cells precisely at the G1 stage as taxol does (Nam et al., 2002). Apart from the anticancer and anti-hyperglycaemic effects, one recent study pointed out that mulberry leaf polyphenols delayed the aging process and regulated fat metabolism in C. elegans (Zheng et al., 2014). Mulberry also plays a protective role against different stresses; for example, it protects pancreatic $\beta$-cells from hydrogen peroxide via an anti-oxidant pathway (Lee et al., 2014).

The diverse activities of mulberry could make it a "wonder plant"; the protective activity of the mulberry could save the non-cancer cells, while killing the cancer cells. To elucidate the MARE's mechanism of action against neuroblastoma, we chose the B103 cell as a model cell line and different molecular pathways were studied with regard to cancer. The results suggest that MARE has a profound cytotoxic effect on B103 cells, but does not possess any toxicity against normal cells. The induction of ROS by MARE treatment damages the mitochondrial membrane potential and its downstream, caspase 3 localization into the nucleus. MARE also regulates FOXO3a translocation in an Akt-dependent manner. Overall, MARE could reduce proliferation and induce cell death by the reduction of Akt and induction of caspase 3 activity, respectively.

\section{MATERIALS AND METHODS}

\section{Reagents}

Dulbecco's Modified Eagle's Medium (DMEM), Fetal Bovine Serum (FBS), and Penicillin/Streptomycin were procured from GIBCO-BRL (USA). Antibodies against PI3K, p-PI3K, Akt, pAkt (Ser473), cleaved caspase-3, Bax, Bcl-2, p-Fox03A (Thr32), Fox03A, and $\beta$-actin were purchased from Cell Signaling Technology (USA) and anti-BIM was purchased from BD Bioscience.

\section{Preparation of extract}

Morus alba root was boiled with $70 \%$ ethanol at $100^{\circ} \mathrm{C}$, and filtered. The filtrate was then concentrated in a desiccator at room temperature. The obtained powder was dissolved in dimethyl sulfoxide (DMSO) before treatment. Four different concentrations were used in the experiment: 5, 10, 15, and 20 $\mu \mathrm{g} / \mathrm{ml}$.

\section{Cell cultures}

Rat B103 neuroblastoma and Rat-2 fibroblast cells were grown at $37^{\circ} \mathrm{C}$ under a humidified and ambient oxygen level in a $\mathrm{CO}_{2}$ incubator. Cells were cultured in DMEM containing $10 \%$ fetal bovine serum (FBS), $50 \mathrm{U} / \mathrm{ml}$ penicillin, and $50 \mu \mathrm{g} / \mathrm{ml}$ streptomycin. The cells were trypsinized before harvest and subcultured.

\section{Cell viability assay}

Cell viability was determined using a cytotoxicity assay kit CCK8 (Dojindo Lab, Japan), which was performed in accordance with the manufacturer's instructions. After treatment, cells were kept for incubation, as required by the experiment. A 96-well microtitre plate reader (Molecular Devices) was used to determine the absorbance at $450 \mathrm{~nm}$.

\section{Morphological analysis}

For the cell morphology experiment, cells were grown in 6-well plate and treated with MARE. After $24 \mathrm{~h}$ of incubation, the cells were examined under a bright-field inverted microscope and digitally imaged.

\section{DAPI staining}

B103 cell were grown on glass cover-slips in a 24-well plate and treated with MARE. After the incubation, cells were fixed with $4 \%$ paraformaldehyde, followed by a wash with PBS. Cells were then stained with DAPI and observed under a DAPI filter by a fluorescence microscope.

\section{Apoptosis analysis}

B103 cells were grown and treated with MARE for $24 \mathrm{~h}$. After incubation, cells were stained with annexin $\mathrm{V}$ and 7-AAD to determine apoptosis; with DCFDA to determine the ROS level; and with rhodamine 123 to measure the mitochondrial membrane potential. Stained cells analysed via flow cytometry (FACS Callibur, BD Bioscience) under their specific filters.

Indirect inter cellular staining: caspase 3 activity assay The caspase- 3 activity assay was performed as per the manufacturer's protocol, using an anti-caspase primary antibody and a FITC-tagged secondary antibody. The fluorescence was determined by a flowcytometer using FL-1H filters. Data were analysed with Cyflogic software.

\section{Immunocytochemistry}

After treatment, cells were fixed with $4 \%$ paraformaldehyde. Following a PBS washing, cells were blocked with bovine serum albumin in PBS at room temperature. Cells were incubated overnight with a primary antibody at $4^{\circ} \mathrm{C}$, and then they were washed with PBS, and incubated with the secondary antibody for $2 \mathrm{~h}$ at room temperature. Following another washing in PBS, the stained cover-slips were mounted and observed under fluorescence microscope.

\section{Western blot}

Cells were grown in a $60 \mathrm{~mm}$ culture dish and treated various concentrations of MARE. After incubation, cells were lysed in a buffer containing $2 \% \mathrm{SDS}, \mathrm{Na}_{3} \mathrm{VO}_{4}$ and protease inhibitor cocktail. Equal amounts of proteins were separated by SDS-PAGE (8-15\% reducing gels), transferred to polyvinylidene difluoride membranes (Millipore), and blocked with 5\% non-fat milk. Membranes were incubated with a specific primary antibody and secondary antibody.

\section{Statistical analysis}

Results were expressed as mean \pm SEM. Statistical significance was analysed by $t$-test using Prism 5 (Graph pad Software). $p<0.05$ was considered significant. All the experiments were repeated thrice and the reported values represent the mean values.

\section{RESULTS}

MARE reduces viability and proliferative potential of B103 cells without affecting Rat-2 cells' viability

To check whether MARE-treatment reduces viability, a cancerous cell line, B103, and a normal cell line, Rat-2, were chosen. Results of a viability assay revealed that the cancer cells' viability gradually reduces between minimal MARE concentrations $(0.5 \mu \mathrm{g} / \mathrm{ml})$ and higher concentrations $(10-20 \mu \mathrm{g} / \mathrm{ml})$, but that the same treatment against normal cells showed that MARE was not cytotoxic towards them (Fig. 1A). At $10 \mu \mathrm{g} / \mathrm{ml}$ of MARE, cells appeared to be growth-arrested, so to check this event, the anti-proliferative activity was further investigated by a CCK8 assay. We found that MARE was able to reduce the proliferation of B103 cells (Fig. 1A). The reduction of growth was promi- 
A

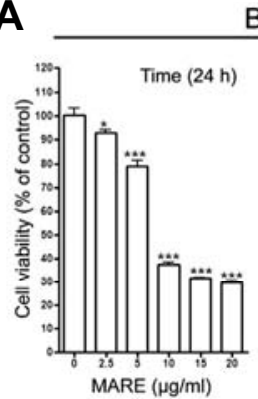

B
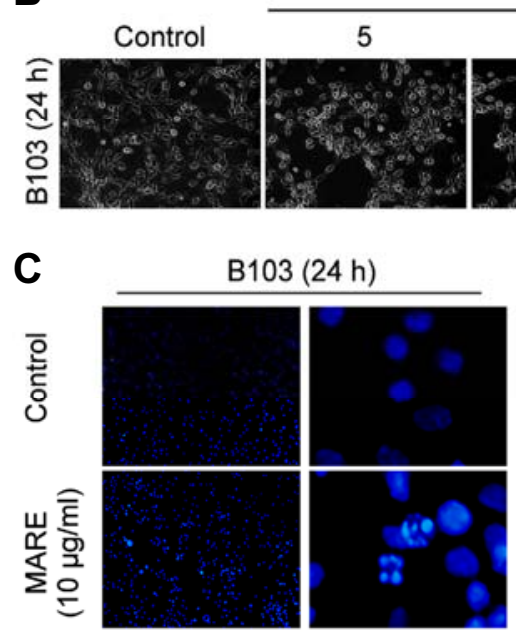

B103

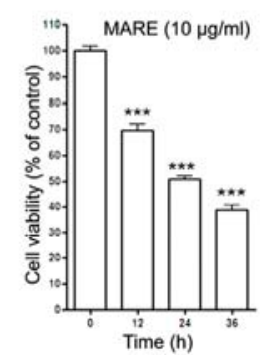

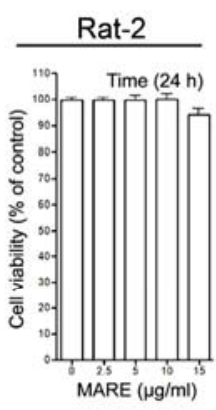

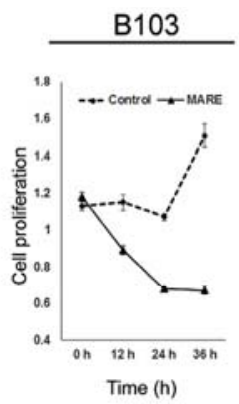

$\operatorname{MARE}(\mu \mathrm{g} / \mathrm{ml})$
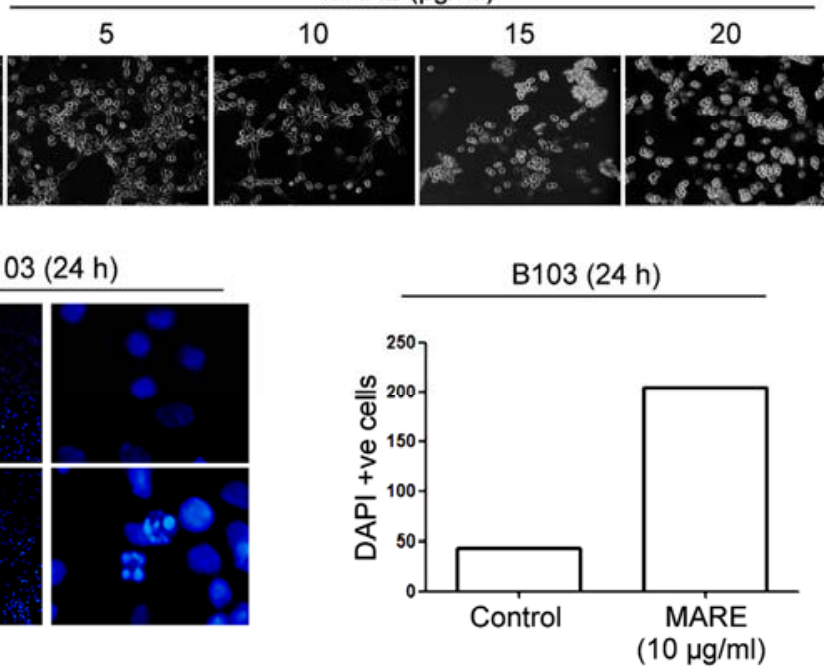

Fig. 1. (A) To evaluate the cytotoxic effects of MARE in B103 rat neuroblastoma cells and Rat-2 rat fibroblast cells, we performed a cell viability assay using a cytotoxicity assay kit (CCK-8). Cells were exposed to different does of MARE (0 to $20 \mu \mathrm{g} / \mathrm{ml}$ ); 10 $\mu \mathrm{g} / \mathrm{ml}$ of MARE was used for the timedependent studies. Data are composed of the mean from three independent experiments in which the activity in the absence of MARE versus in the presence of MARE is significantly different $\left(\mathrm{n}=4,{ }^{*} P<0.05,{ }^{* *}<\right.$ $0.01,{ }^{* * *}<0.001$ ). (B) Cells were treated with different doses of MARE (0 to 20 $\mu \mathrm{g} / \mathrm{ml})$. After $24 \mathrm{~h}$, they were digitally photographed (200X). The photographs show rounding and cell detachment from the culture surface when treated with MARE (C) Cells were grown on cover-slips in 24-well plates and treated without MARE (control) and with $10 \mu \mathrm{g} / \mathrm{ml}$ MARE. After $24 \mathrm{~h}$ incubation, cells were fixed and washed and stained with DAPI. There is a clear indication of condensed DNA in the MAREtreated cells. DAPI-positive cells were quantified. nent after $12 \mathrm{~h}$ of drug treatment and proliferation gradually decreased as the incubation time increased in comparison with the control (vehicle/DMSO-treated) sets. Combining both the events, we suggest that MARE treatment could result in cytotoxicity, particularly toward neuroblastoma cells, while reducing their proliferation.

\section{MARE induces differentiated cellular and nuclear morphology in B103 cells}

The induction of cytotoxicity could bring upon morphological changes in the cells. To check for potential morphological alteration, different doses of MARE were applied to B103 cells and observed microscopically. Microscopic images of MARE-treated cells showed significant morphological changes as compared to the control cells. The treated cells showed a rounding of the cellular structure with the 'bleb' formation (Fig. 1B). The nuclear morphology was observed by DAPI staining. The result of fluorescent microscopic images clearly demonstrated the condensation of nuclear chromatin with the pyknotic bodies formation (DAPI positive cells) (Fig. 1C). The observation reveals that the MARE cytotoxicity in B103 cells leads to morphological changes within the cells, potentially a hallmark of apoptosis.

MARE treatment produces early apoptotic cells by involving caspase 3

A change in morphology with the induction of cytotoxicity was prominent when B103 cells were treated with MARE, representing the hallmark of apoptosis. We examined whether MARE-treated cell death was apoptotic. To do so, we stained the cells with annexin $\mathrm{V}$ and 7AAD. Annexin $\mathrm{V}$ binds with the phosphatidylserine (PS), under normal physiological conditions PS located in the inner leaflet of the plasma membrane. Upon induction of apoptosis, PS loses asymmetric distribution across the phospholipid bilayer and is translocated to the extracellular membrane leaflet. Once on the outer surface of the membrane, PS can be detected by fluorescently labelled Annexin V. In early-stage apoptosis, the plasma membrane excludes viability dye like 7-AAD. These cells will stain with Annexin $\mathrm{V}$ but not a viability dye, thus distinguishing cells in early apoptosis. In our result, flow cytometry data clearly indicated that there was a distinct formation of a population of cells in the annexin $\mathrm{V}$ positive-7AAD negative quadrant after MARE treatment (Fig. 2A). The population shift was greater toward that quadrant at the higher doses of MARE (Fig. 2B). The result revealed that the cell death caused by MARE was early apoptosis.

To further investigate the possible signalling pathway associated with the apoptosis, we checked the activity, expression, and the localization of the caspase 3 protein by FACS, immunoblotting, and microscopy. Flow cytometry data suggested that MARE treatment increased caspase 3 activity, which was down-regulated when co-treated with a caspase 3 inhibitor (Z-DEVD-FMK) (Fig. 3A). The results from a western blot also suggested that there was an up-regulation of expression in the treatment groups ( 2.3 to $\sim 2.4$ folds) than in the control cells (Fig. 3B). Fluorescent microscopic images revealed that activated caspase 3 protein was localized in the nucleus of the cancer cells (Fig. $3 \mathrm{C}$ ). By combining all of these results, we could predict that MARE treatment activates caspase 
A

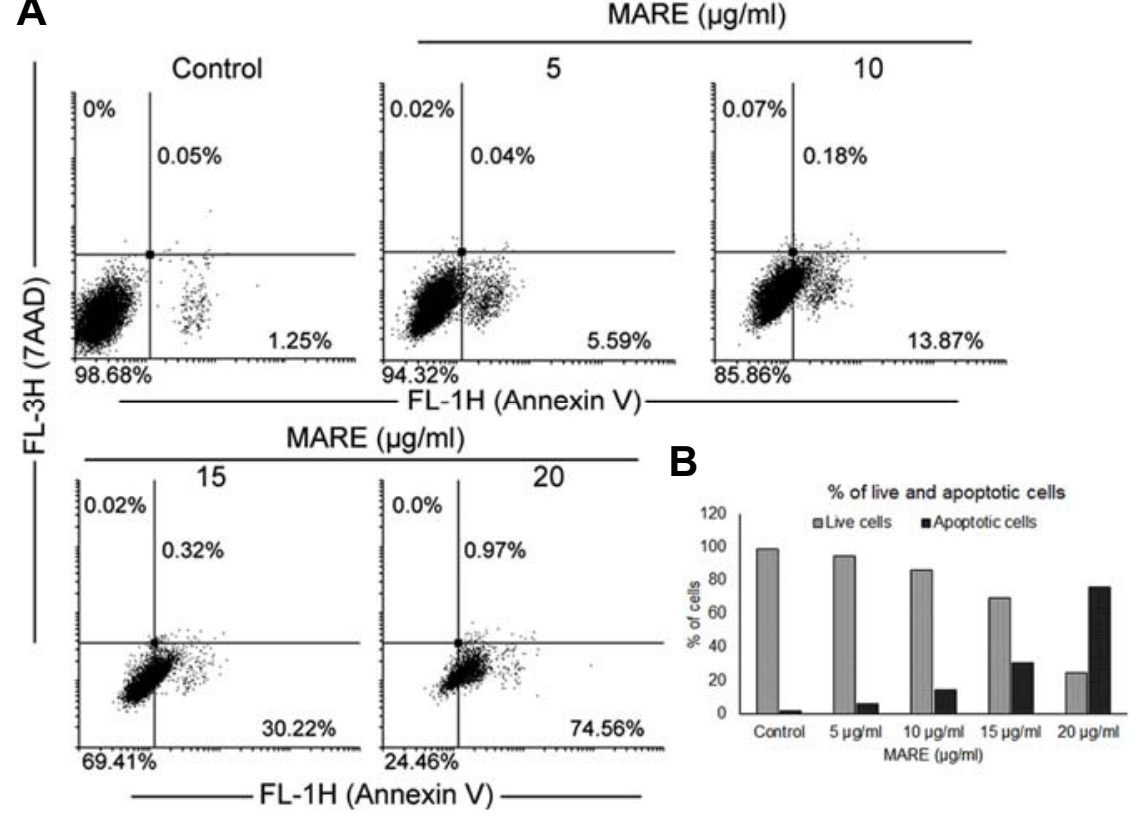

Fig. 2. (A) Cells were grown and treated with various concentrations of MARE for $24 \mathrm{~h}$. The cells were stained with Annexin $V$ and 7-AAD and analyzed through flow cytometry. (B) Annexin Vpositive cells were quantified; the results are shown in the graph.
A

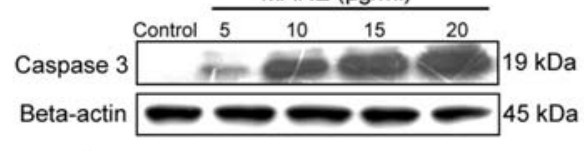

C

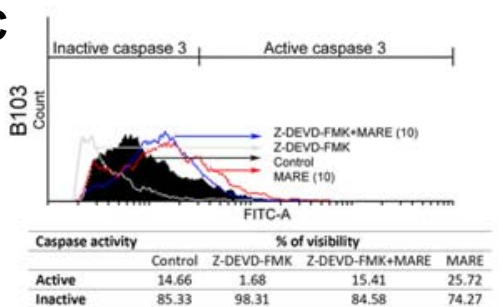

B

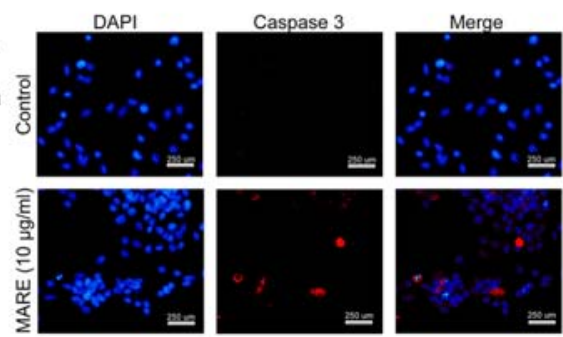

Fig. 3. (A) MARE-treated cells were lysed and subjected to $15 \%$ SDS-PAGE. Caspase-3 protein was detected by western blotting. $\beta$-actin was used a loading control. (B) Cells were grown on coverslips in 24-well plates. Cells were treated with 0 (control) and $10 \mu \mathrm{g} / \mathrm{ml}$ of MARE for $24 \mathrm{~h}$. After treatment, cells were fixed with 4\% PFA and were incubated overnight with the primary antibody (cleaved caspase-3) at $4^{\circ} \mathrm{C}$. Cells were stained with a secondary antibody and observed under fluoresce microscope (200X). Results indicated there is an increase in the activity of caspase 3 in the cells' nucleus (C). The activity of caspase-3 in MARE-treated cells was evaluated by flow cytometry using a caspase-3 antibody by indirect staining and the results indicate that, upon co-treatment of caspase-3 inhibitor (Z-DEVD-FMK) with MARE, the ability of MARE to activate caspase-3 is reduced.
3, which induces DNA damage and apoptosis.

MARE treatment generates reactive oxygen species in B103 cells

To examine the possible events in the induction of the apoptotic death pathway, we evaluated ROS generation in B103 cells after MARE treatment. ROS is a novel tool used to combat cancer, and it could create an apoptotic condition in the cancer cells when a threshold tolerance limit is crossed. Treated and control cells were stained with DCFDA and counted via flow cytometry. The control cells had the smallest amount of ROS in them, but the amount of ROS increased in a dose-dependent manner with MARE administration (Fig. 4A). This observation shows that the increase in ROS inside the cells may damage the mitochondria membrane, depolarizing it to induce apoptosis.

MARE treatment depolarizes MMP and upsets the Bcl-2/Bax ratio

To check whether MARE treatment could depolarize the mito- chondria membrane, we stained B103 cells with Rhodamine 123 and estimated mitochondrial membrane depolarization with flow cytometry. We found that, with MARE treatment, mitochondrial membrane depolarization increased in a dosedependent manner (Fig. 4B). To evaluate the proteins responsible for this process, we further verified the expression of different Bcl-2 family proteins. With MARE treatment, antiapoptotic Bcl-2 expression decreased with an increase in Bax expression, and thus, the ratio between $\mathrm{Bcl}-2$ and Bax was disrupted (Fig. 4C). Consequently, we now propose that MARE possesses the capacity to induce apoptosis in neuroblastoma by inducing ROS and MMP depolarization, the process which results in caspase 3 activation.

MARE induced ROS plays the central role in controlling apoptosis and proliferation

To investigate the involvement of MARE induced ROS in controlling apoptosis and proliferation, we treated MARE on B103 cells in presence or absence of NAC (N-acetyl cysteine) and 
A

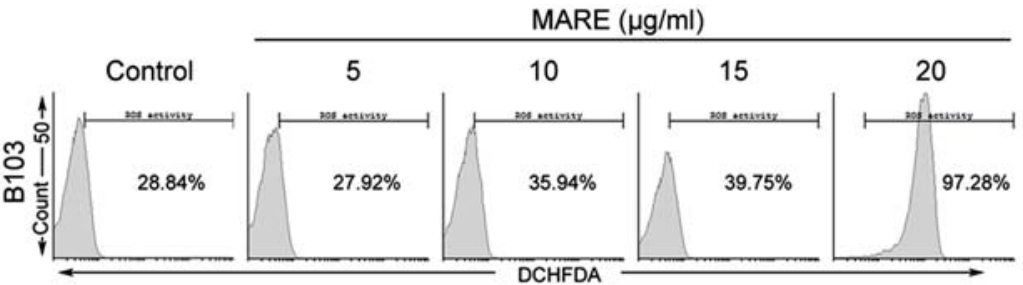

B

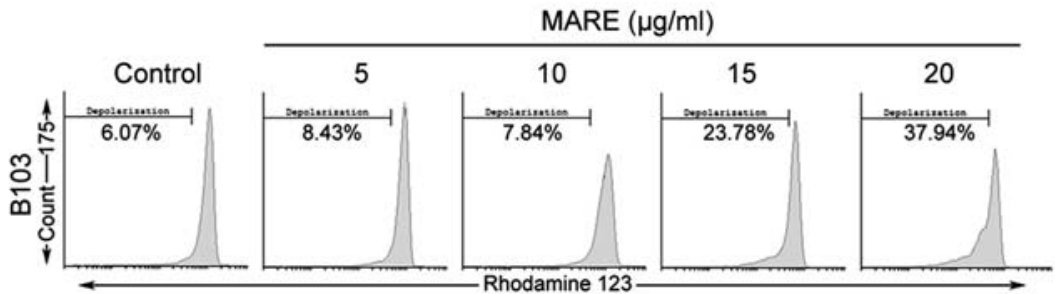

C

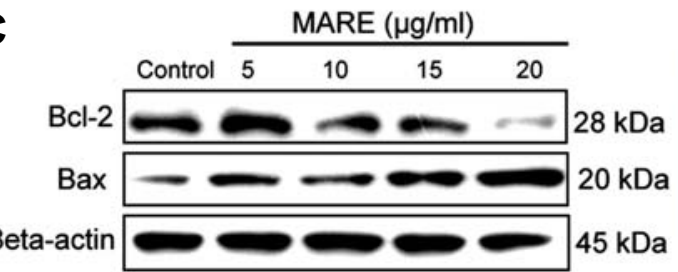

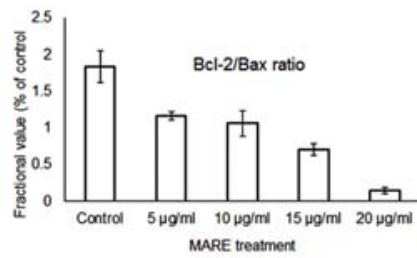

Fig. 4. (A and $B$ ) Cells was grown and treated various concentrations of MARE for $24 \mathrm{~h}$. We stained the cells with DCHFDA and Rhodamine123 to estimate ROS and MMP, respectively. Analyzed through flow cytometry, the results show MARE has the capability to accumulate ROS inside the cells and depolarize the mitochondria membrane. (C) MARE-treated cells were lysed and subjected to $12-15 \%$ SDS-PAGE. Bax and $\mathrm{Bcl}-2$ proteins were detected by western blotting. $\beta$-actin was used a loading control. The Bcl-2/Bax ratio was quantified using ImageJ software. preformed annexin $\mathrm{V}$ assay, cell viability assay and proliferation assay. We found that in MARE and NAC co-treated samples, the formation of annexin $\mathrm{V}$ positive cells downed to the normal level (Fig. 5A). The similar kind of result was observed in the viability assay. We found that in NAC/MARE co-treated B103 cells, viability was increased further and became at per with the MANE-untreated B103 cells (Fig. 5B). In the proliferation assay result we found that the co-treatment of NAC with MARE treatment resist the effectiveness of MARE on proliferation (Fig. 5C). By combining all the findings, we can proposed that MARE induced ROS accumulation further controls the process of apoptosis induction and proliferation reduction.

\section{MARE treatment modulates proliferation and transcription markers}

Akt is constitutively dynamic in most cancer cells, and it enhances cell proliferation. In order to understand the association between PI3K/Akt and MARE-induced growth inhibition, here, we determined the expression of protein phosphorylation of PI3K and Akt in cells exposed to MARE. MARE resulted in slight inhibition of phosphorylation of PI3K (Tyr458) and completely blocked phosphorylation of PI3K (Tyr199) in B103 cells (Fig. 6A) and relatively marked inhibition of Akt phosphorylation (Fig. 6A). MARE had no effect on total PI3K and Akt expression (Fig. 6A). These results suggested that MARE-mediated Akt signaling inhibition may contribute to growth inhibition of B103 cells. However, the phosphorylation of $\mathrm{FOXO} 3$ a proteins was analysed using western blotting. MARE inhibited the phosphorylation of FOXO3a protein. Conversely, MARE-treatment has dose-dependently increased on the total protein of FOXO3a expression (Fig. 6A). These results suggested that MARE inhibited growth by regulating $\mathrm{FOXO} a$ and suggesting FOXO3a may be associated with kinase activity. At the same time, MARE caused a very clear upregulation of Bim levels in the B103 cells (Fig. 6A). Further, we checked the localization of FOXO3a with fluorescence microscopy and the results indicated that FOXO3a was located inside the nucleus (Fig. 6B). Therefore, the translocation of FOXO factors into the nucleus from cytoplasm activates apoptosis signals through upregulating BIM activity (Myatt and Lam, 2007). Taken together, these results suggest that FOXO3a may be a novel downstream way of MARE-treatment against B103 cells.

\section{DISCUSSION}

The present study reports the anti-cancer effects of MARE against neuroblastoma. We observed that MARE elicited the upregulation of caspase 3 activity and the down-regulation of Akt and FOXO3a phosphorylation. The blocking of growth inductive signals and induction of ROS by the drug induced a rapid apoptotic response in the neuroblastoma-B103 cells. These included morphological changes that occurred within 24 $\mathrm{h}$ of MARE administration, with progressive detachment of cells containing condensed and fragmented chromatin. The results also suggest that the viability of cancer cells is reduced in a dose-dependent manner. MARE's interference with the mitochondria membrane and caspase-mediated apoptosis through a disruption of the Bcl-2/Bax ratio further confirms MARE's apoptosis-inducing capability in neuroblastoma cells. MARE shows a relative low toxicity toward non-cancerous cells, which suggests that MARE could be an effective and safe anticancer drug.

Although a study on multi-drug resistant breast cancer and leukaemia with MARE indicated that MARE plays an effective role in inducing the JNK pathway (Choi et al., 2013), resulting in the induction of apoptosis, but there is a lack of knowledge about the activities of MARE on neuroblastoma. The purpose of this study was to investigate the probable mechanisms of MARE's toxicity against neuroblastoma cells.

The mulberry plant has been reported to be rich in flavonoids 
A
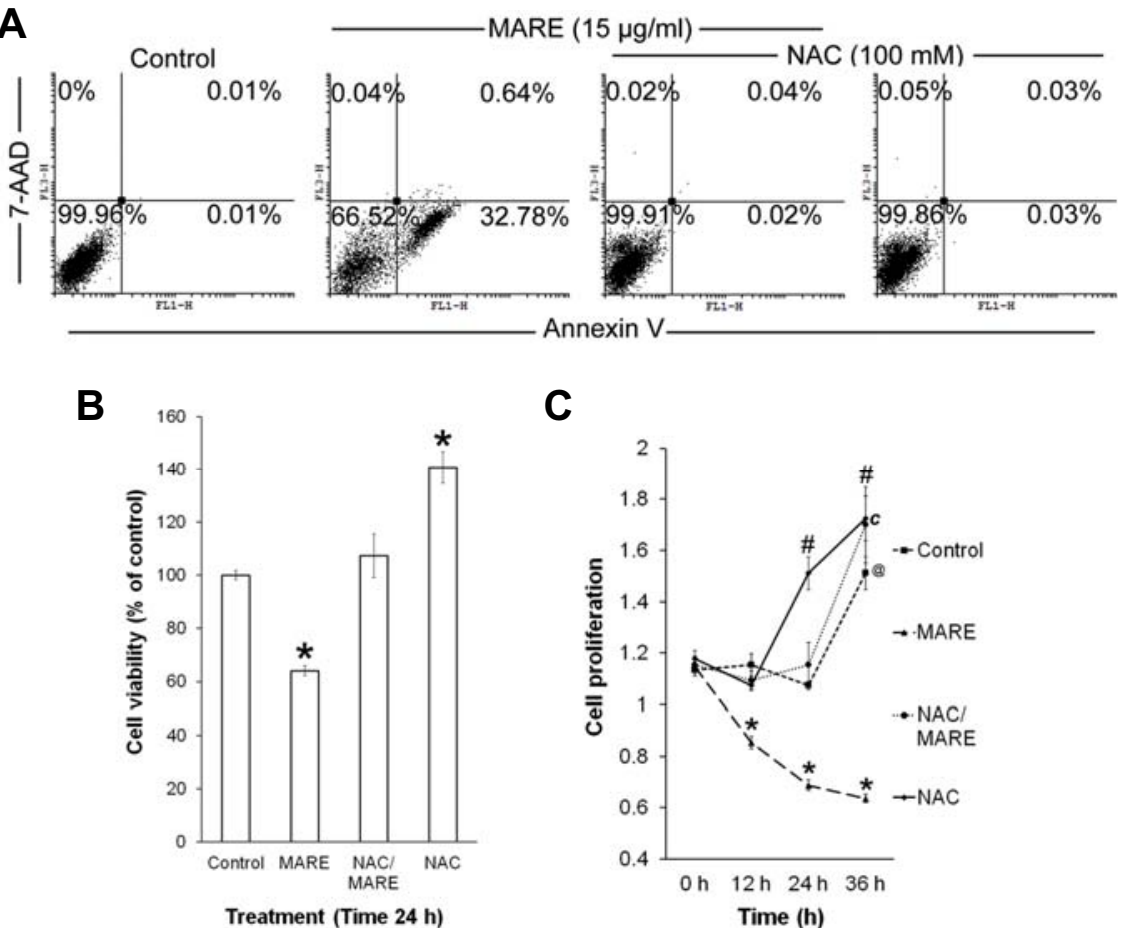

A

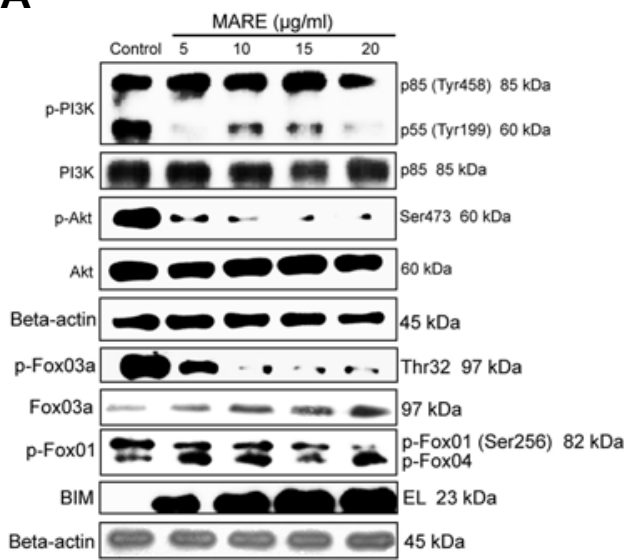

B
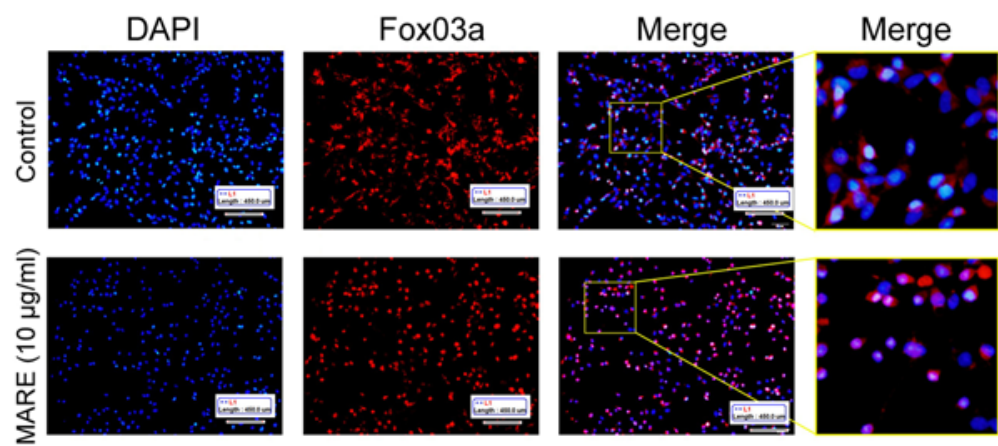

Fig. 6. (A) MARE-treated cells were lysed and subjected to 8-15\% SDS-PAGE. PI3K, p-PI3K, Akt, p-Akt (ser473), FOXO3a, p-FOXO3a (Thr32), p-FOXO1 (ser256), and Bim proteins were detected by western blotting. $\beta$-actin was used a loading control. (B) Cells were grown on cover-slips in 24-well plates. Cells were treated with 0 and $10 \mu \mathrm{g} / \mathrm{ml}$ of MARE for $24 \mathrm{~h}$. After treatment, cells were fixed with $4 \%$ PFA and were incubated overnight with the primary antibody (FOXO3a) at $4^{\circ} \mathrm{C}$. Cells were stained with the secondary antibody and observed under a fluoresce microscope (200X).

that have different biological activities, including its anti-oxidant capacity (Zheng et al., 2014). In this study, MARE treatment of B103 cells was found to stimulate ROS accumulation and further depolarize the MMP. Studies on ROS reveal that its exposure beyond the tolerated limit usually pushes cancer cells towards apoptosis by disrupting mitochondrial integrity (Huang et al., 2014). Recent reports have demonstrated dramatic alterations in mitochondrial morphology during the early stages of apoptotic cell death, including a fragmentation of the network and the remodelling of the cristae (Suen et al., 2008). Surpris- ingly, proteins discovered to control mitochondrial morphology appear to also participate in apoptosis and proteins associated with the regulation of apoptosis have been shown to affect mitochondrial ultra-structure (Karbowski and Youle, 2003). Bax translocation to the mitochondrial membrane creates a pore, which otherwise depolarize the mitochondria membrane potential (Henry-Mowatt et al., 2004).

Members of the Bcl-2 family proteins are known to play a critical regulatory role in the apoptosis pathway (Martinou and Youle, 2011). Bcl-2 has been shown to form a heterodimer with 


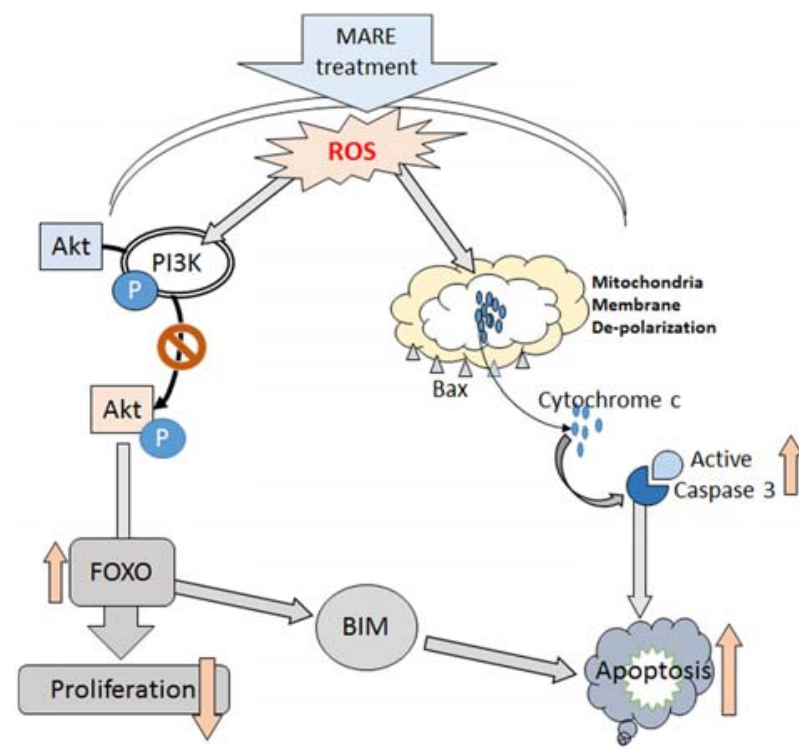

Fig. 7. Schematic representation: Action of MARE on neuroblastoma cells

pro-apoptotic Bax, a union which may neutralize its proapoptotic effect (Moldoveanu et al., 2014). Therefore, an alteration in the levels of Bcl-2 and Bax could be a decisive factor in the induction of apoptotic conditions in cells. In our study, Bax expression increased while Bcl-2 expression decreased with MARE treatment, thus disrupting the balance between them, which is a major event in the initiation of apoptosis involving the mitochondria. Through this distorted mitochondrial membrane, cytochrome $\mathrm{c}$ is released out to the cytosol of the cells (Chipuk et al., 2006) and initiates the formation of an apoptosome along with the adopter molecule Apaf-1 and other pro-caspase molecules like caspase- 9 and 3 (Tait and Green, 2010). Downstream, activated caspase 3 induces chromatin condensation and degradation through nick formation (Kitazumi and Tsukahara, 2011). The activated caspase 3 and presence of DAPI-positive cells in the MARE treated samples, is a clear indicator of the induction of apoptosis involving mitochondria and caspase 3 .

The anti-neoplastic effect of a drug could also diminish the propagative potential of cancer cells along with the reduction of different growth signals and prevention of specific transcriptions (Hanahan and Weinberg, 2011). Akt and FOXO-family proteins are two distinct agents that control cancer growth and metastasis (Boreddy et al., 2011). Class I PI3K protein is responsible to control the process of Akt phosphorylation and activation. The class $1 \mathrm{PI} 3 \mathrm{~K}$ is composed of a heterodimer between $\mathrm{p} 110$ catalytic subunit and a p85 regulatory subunit and there are different variants of the p85 regulatory subunit, one among them is p55. Phosphorylation on p55 regulatory region alternatively controls the activity of pl3K enzyme. Reduction in phosphorylation on p55 region of PI3K in tum indicated the conquest of its activity (Carpenter et al., 1990). The inhibitory effects of MARE on neuroblastoma growth not only resulted from caspase 3 activation, but also through the de-activation of PI3K and Akt. Phosphorylation at different serine and threonine sites of Akt contributes to its activation (Wang and Basson, 2011). Phosphorylation at Ser473 of Akt by $\mathrm{PI} 3 \mathrm{~K}$ is responsible for the activation and membrane attachment of Akt, causing the acceleration of downstream growth signals
(Wang and Basson, 2011). MARE treatment reduces the phosphorylation events of Akt protein, and thus rendering it irrelevant for growth signal induction.

Proliferation via different growth signals involves the process of transcription and activated transcription factors. FOXO3a, a known transcription factor, is a direct downstream molecule of Akt (Zhang et al., 2011). Transcriptional activity and localization of FOXO3a is regulated by Akt. Akt-mediated phosphorylation of FOXO3a is located in the cytoplasm. However, inactivation of Akt leads to the de-phosphorylation of FOXO3, activating it (Brunet et al., 1999). Accordingly, FOXO3a translocate into the nucleus and where it increases the transcriptional activity of its target gene, the apoptotic protein, Bim (Myatt and Lam, 2007). So, the activation of $\mathrm{FOXO} 3$ a triggers apoptosis through its regulation of Bim activity. MARE treatment on neuroblastoma cells supports the same phenomenon (Fig. 7).

In conclusion, we explored the anticancer potential of MARE against neuroblastoma, finding evidence that it possesses considerable anticancer potential in addition to its anti-diabetic properties. We found that the apoptotic pathway linked to the mitochondria-dependent caspase route, through caspase 3 activation, resulting in the death of the cancer cells occurred with the administration of MARE. De-phosphorylation of Akt and FOXO3a with MARE treatment makes the cancer cells anti-proliferative. The dual role played by this drug could be of interest in the design and formulation of specialized drugs.

\section{ACKNOWLEDGMENTS}

This research was supported by Basic Science Research Program through the National Research Foundation of Korea(NRF) funded by the Ministry of Education(NRF-20090094071) and by Hallym University (HRF-201501-020), South Korea.

\section{REFERENCES}

Bengmark, S., Mesa, M.D., and Gil, A. (2009). Plant-derived health: the effects of turmeric and curcuminoids. Nutr. Hosp. 24, 273281.

Boreddy, S.R., Pramanik, K.C., and Srivastava, S.K. (2011). Pancreatic tumor suppression by benzyl isothiocyanate is associated with inhibition of PIJK/AKT/FOXO pathway. Clin. Cancer Res. 17, 1784-1795.

Brunet, A., Bonni, A., Zigmond, M.J., Lin, M.Z., Juo, P., Hu, L.S. Anderson, M.J., Arden, K.C., Blenis, J., and Greenberg, M.E. (1999). Akt promotes cell survival by phosphorylating and inhibiting a Forkhead transcription factor. Cell 96, 857-868.

Carpenter, C.L., Duckworth, B.C., Auger, K.R., Cohen, B. Schaffhausen, B.S., and Cantley, L.C. (1990). Purification and characterization of phosphoinositide 3-kinase from rat liver. J. Biol. Chem. 265, 19704-19711.

Chakraborty, D., Ghosh, S., Bishayee, K., Mukherjee, A., Sikdar, S., and Khuda-Bukhsh, A.R. (2013). Antihyperglycemic drug Gymnema sylvestre also shows anticancer potentials in human melanoma A375 cells via reactive oxygen species generation and mitochondria-dependent caspase pathway. Integr. Cancer Ther. 12, 433-441.

Chipuk, J.E., Bouchier-Hayes, L., and Green, D.R. (2006). Mitochondrial outer membrane permeabilization during apoptosis: the innocent bystander scenario. Cell Death Differ. 13, 1396-1402.

Choi, Y.K., Cho, S.G., Choi, H.S., Woo, S.M., Yun, Y.J., Shin, Y.C., and Ko, S.G. (2013). JNK1/2 Activation by an Extract from the Roots of Morus alba L. Reduces the Viability of MultidrugResistant MCF-7/Dox Cells by Inhibiting YB-1-Dependent MDR1 Expression. Evid. Based Complement. Alternat. Med. 2013, 741985

Eo, H.J., Park, J.H., Park, G.H., Lee, M.H., Lee, J.R., Koo, J.S., and Jeong, J.B. (2014). Anti-inflammatory and anti-cancer activity of mulberry (Morus alba L.) root bark. BMC. Complement. Altern. 
Med. 14, 200.

Fish, J.D., and Grupp, S.A. (2008). Stem cell transplantation for neuroblastoma. Bone Marrow Transplant. 41, 159-165.

Friedman, G.K., and Castleberry, R.P. (2007). Changing trends of research and treatment in infant neuroblastoma. Pediatr. Blood Cancer 49, 1060-1065.

Gupta, R.K., and Patel, A.K. (2013). Do the health claims made for Morinda citrifolia (Noni) harmonize with current scientific knowledge and evaluation of its biological effects. Asian Pac. J. Cancer Prev. 14, 4495-4499.

Haase, G.M., Perez, C., and Atkinson, J.B. (1999). Current aspects of biology, risk assessment, and treatment of neuroblastoma. Semin. Surg. Oncol. 16, 91-104.

Hanahan, D., and Weinberg, R.A. (2011). Hallmarks of cancer: the next generation. Cell 144, 646-674.

Henry-Mowatt, J., Dive, C., Martinou, J.C., and James, D. (2004). Role of mitochondrial membrane permeabilization in apoptosis and cancer. Oncogene 23, 2850-2860.

Huang, H.W., Chung, Y.A., Chang, H.S., Tang, J.Y., Chen, I.S., and Chang, H.W. (2014). Antiproliferative effects of methanolic extracts of Cryptocarya concinna Hance roots on oral cancer Ca9-22 and CAL 27 cell lines involving apoptosis, ROS induction, and mitochondrial depolarization. Scientific World Journal 2014, 180462.

Johnson, E., Dean, S.M., and Sondel, P.M. (2007). Antibody-based immunotherapy in high-risk neuroblastoma. Expert Rev. Mol. Med. 9, 1-21.

Karbowski, M., and Youle, R.J. (2003). Dynamics of mitochondrial morphology in healthy cells and during apoptosis. Cell Death Differ. 10, 870-880.

Kikuchi, T., Nihei, M., Nagai, H., Fukushi, H., Tabata, K., Suzuki, T., and Akihisa, T. (2010). Albanol A from the root bark of Morus alba L. induces apoptotic cell death in HL60 human leukemia cell line. Chem. Pharm. Bull. 58, 568-571.

Kitazumi, I., and Tsukahara, M. (2011). Regulation of DNA fragmentation: the role of caspases and phosphorylation. FEBS J. $278,427-441$

Lastowska, M., Cullinane, C., Variend, S., Cotterill, S., Bown, N., O'Neill, S., Mazzocco, K., Roberts, P., Nicholson, J., Ellershaw, C., et al. (2001). Comprehensive genetic and histopathologic study reveals three types of neuroblastoma tumors. J. Clin. Oncol. 19, 3080-3090.

Lee, J.S., Kim, Y.R., Park, J.M., Ha, S.J., Kim, Y.E., Baek, N.I., and Hong, E.K. (2014). Mulberry fruit extract protects pancreatic beta-cells against hydrogen peroxide-induced apoptosis via antioxidative activity. Molecules 19, 8904-8915.

Mantena, S.K., Sharma, S.D., and Katiyar, S.K. (2006). Berberine, a natural product, induces G1-phase cell cycle arrest and caspase-3-dependent apoptosis in human prostate carcinoma cells. Mol. Cancer Ther. 5, 296-308.

Martinou, J.C., and Youle, R.J. (2011). Mitochondria in apoptosis: Bcl-2 family members and mitochondrial dynamics. Dev. Cell 21 , 92-101.

Moldoveanu, T., Follis, A.V., Kriwacki, R.W., and Green, D.R. (2014). Many players in BCL-2 family affairs. Trends Biochem. Sci. 39, 101-111.

Mondal, S., Bandyopadhyay, S., Ghosh, M.K., Mukhopadhyay, S., Roy, S., and Mandal, C. (2012). Natural products: promising resources for cancer drug discovery. Anticancer Agents Med. Chem. 12, 49-75.

Myatt, S.S., and Lam, E.W. (2007). The emerging roles of forkhead box (Fox) proteins in cancer. Nat. Rev. Cancer 7, 847-859.

Nam, S.Y., Yi, H.K., Lee, J.C., Kim, J.C., Song, C.H., Park, J.W. Lee, D.Y., Kim, J.S., and Hwang, P.H. (2002). Cortex mon extract induces cancer cell apoptosis through inhibition of microtubule assembly. Arch. Pharm. Res. 25, 191-196.

Nuchtern, J.G. (2006). Perinatal neuroblastoma. Semin. Pediatr. Surg. 15, 10-16.

Ren, C., Zhang, Y., Cui, W., Lu, G., Wang, Y., Gao, H., Huang, L., and $\mathrm{Mu}, \mathrm{Z}$. (2015). A polysaccharide extract of mulberry leaf ameliorates hepatic glucose metabolism and insulin signaling in rats with type 2 diabetes induced by high fat-diet and streptozotocin. Int. J. Biol. Macromol. 72, 951-959.

Smejkal, K., Svacinová, J., Slapetová, T., Schneiderová, K., Dall'acqua, S., Innocenti, G., Závalová, V., Kollár, P., Chudík, S., Marek, R., et al. (2010). Cytotoxic activities of several geranylsubstituted flavanones. J. Nat. Prod. 73, 568-572.

Suen, D.F., Norris, K.L., and Youle, R.J. (2008). Mitochondrial dynamics and apoptosis. Genes Dev. 22, 1577-1590.

Tait, S.W., and Green, D.R. (2010). Mitochondria and cell death: outer membrane permeabilization and beyond. Nat. Rev. Mol. Cell Biol. 11, 621-632.

Wang, S., and Basson, M.D. (2011). Protein kinase B/AKT and focal adhesion kinase: two close signaling partners in cancer. Anticancer Agents Med. Chem. 11, 993-1002.

Wang, S., Fang, M., Ma, Y.L., and Zhang, Y.Q. (2014). Preparation of the Branch Bark Ethanol Extract in Mulberry Morus alba, Its Antioxidation, and Antihyperglycemic Activity In Vivo. Evid. Based Complement. Alternat. Med. 2014, 569652.

Zhang, X., Tang, N., Hadden, T.J., and Rishi, A.K. (2011). Akt, FoxO and regulation of apoptosis. Biochim. Biophys. Acta 1813, 1978-1986.

Zheng, S., Liao, S., Zou, Y., Qu, Z., Shen, W., and Shi, Y. (2014). Mulberry leaf polyphenols delay aging and regulate fat metabolism via the germline signaling pathway in Caenorhabditis elegans. Age (Dordr) 36, 9719 